Military Technical College Kobry El-Kobbah Cairo, Egypt

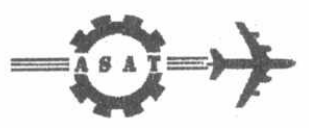

$10^{\text {th }}$ International Conference On Aerospace Sciences\& Aviation Technology

\title{
MODELING OF MICROSCALE SOLID PROPELLANT COMBUSTION
}

\author{
HEGAB ${ }^{1 *}$ A. M.
}

\begin{abstract}
The numerical procedure for the burning of Ammonium Perchlorate (AP) with a Fuel-Binder (Hydroxyl Terminated Polybutadience HTPB) heterogeneous propellant is presented. This model accounts for the one-step reaction mechanism for the primary diffusion flame between the decomposition products of the Binder $(B)$ and the oxidizer $\mathrm{AP}$ and allowed for the complete coupling between the gas-phase physics, the condensed-phase physics, and the unsteady non-uniform regression of the propellant surface. The parameters used in this model are fitted to experimental data for the combustion of AP/Binder. The propagation of the unsteady non-planer regression surface is described, using the Essentially-Non-Oscillatory (ENO) scheme with the aid of the level set strategy. The Alternating-Direction-Implicit (ADI) solver is employed to solve the full Navier-Stokes equations in the gas phase. The results show the effect of various parameters on the surface propagation speed, flame structure, and the burning surface geometry. A comparison between the computational and experimental results is presented.
\end{abstract}

\section{KEY WORDS}

Composite propellant, solid particles, sandwich propellant, AP/HTPB.

\footnotetext{
1* Lecturer, Dept. of Mechanical Power Engineering, Faculty of Engineering, Menoufia University, Shebin Elkom, Egypt.
} 


\section{NOMENCLATURE}

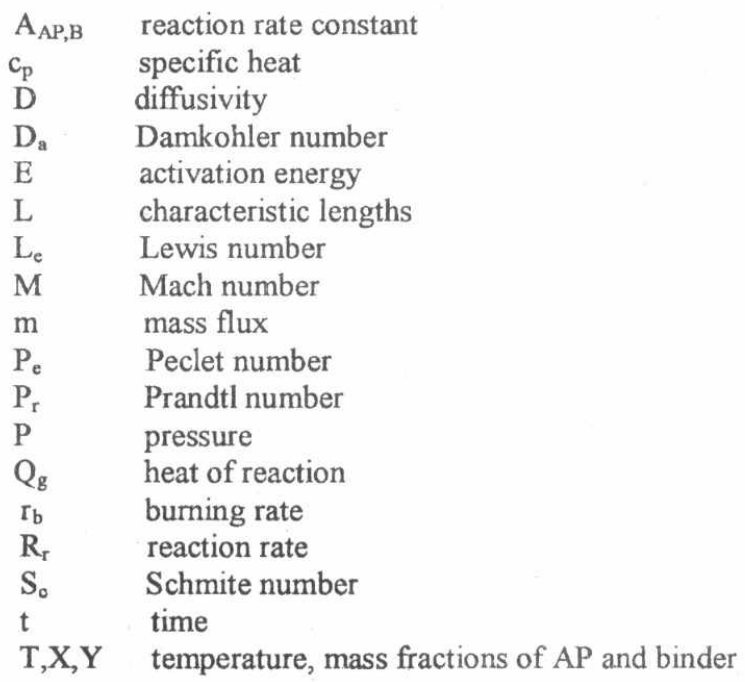

\section{GREEK SYMBOLS}

$\beta \quad$ AP/binder stoichiometric ratio

$\eta \quad$ surface function

$\lambda$ heat conductivity

$v \quad$ fractional binder thickness

$\rho \quad$ density

$\phi \quad$ equivalence ratio

$\psi \quad$ function negative in binder, positive in AP

\section{SUBSCRIPTS}

$\begin{array}{ll}\text { AP } & \text { ammonium perclorate } \\ \text { B } & \text { binder } \\ \text { g } & \text { gas phase } \\ \text { S } & \text { solid phases }\end{array}$

\section{INTRODUCTION}

The complex flame structure that is generated by burning of a heterogeneous solid rocket propellant is proposed by Beckstead, Derr, and Price (the BDP model [1]), as shown schematically in Fig.1. Three separate flames can be identified in the gas phase. 
1. a primary flame between the decomposition products of the binder and the oxidizer, 2 . a premixed oxidizer flame, and 3. a final diffusion flame between the products of the other two flames. In spite of the BDP model is one-dimensional and necessarily omits or fails to properly account for important physics, but attempts to account for many of the significant feature of the combustion field. The influence of this work (published in 1970) still endures [2], and 1D models are still used [3]. Several improvements to BDP model of steady-state burning have been conducted. Lee et. al. [4] presented a modified picture for the flame structure for AP-Binder-AP sandwich as in Fig.2. This sketch show the principles of the combustion zone, in which the oxidizer-fuel flames consists of a leading-Edge Flame (LEF) that stands in the mixing region of the oxidizer and fuel vapors, and a diffusion flame that trails from the LEF up to a point where the fuel vapor is all consumed. The LEF is a region of very high heat release as compared to the rest of the diffusion flames and contributes most of the heat transfer back of the propellant surface. This edge occurs because the diffusion flame can not extend all the way to the surface, the temperature there being too low.

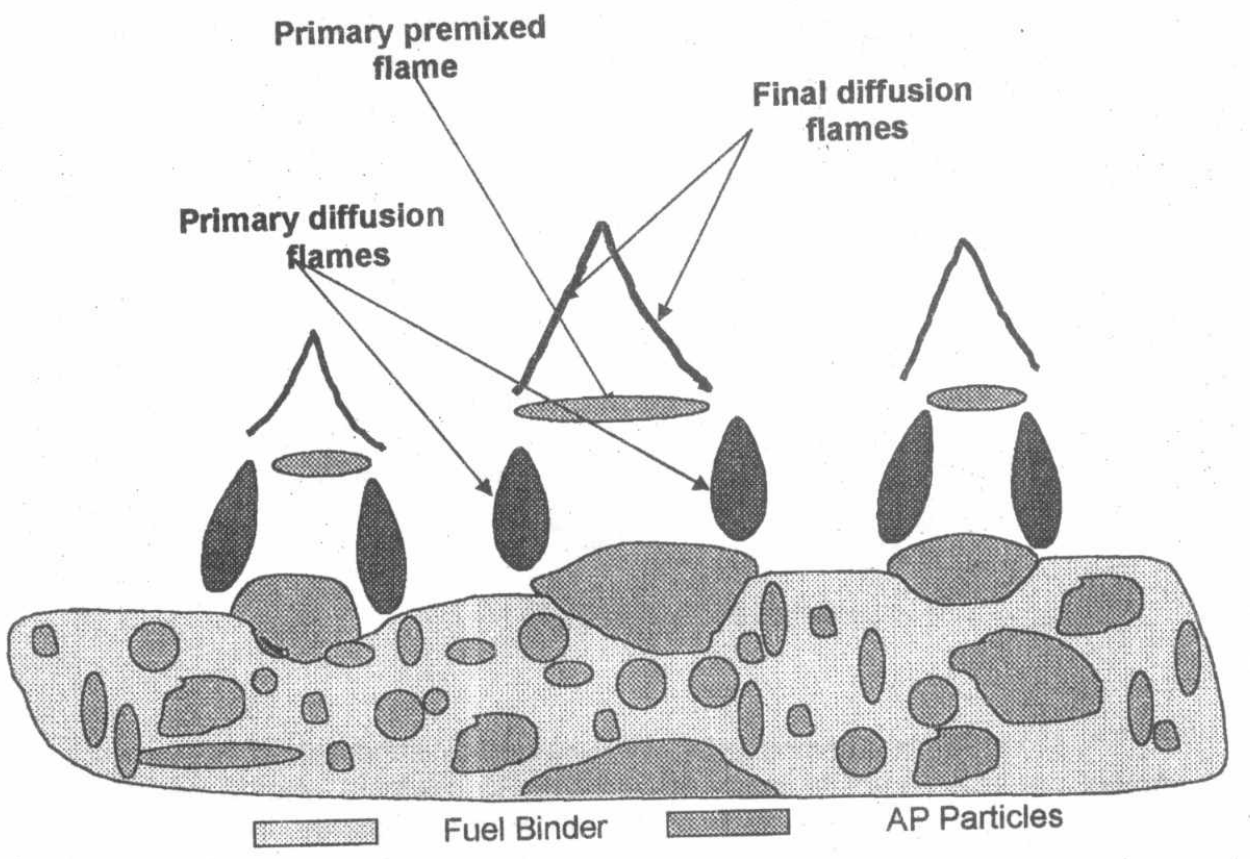

Figure 1: Flame Structure of AP/Binder Composed Propellant Proposed by Beckstead, Derr, and Price [1].

The theoretical studies for the combustion of heterogeneous solid rocket propellant have faced a lot of difficulties because of the chemical and physical complexity of the propellant and the microscopic scale of the combustion zone. Therefore, few experimental studies have been performed for the simplest model of the combustion of 
Ammonium Perchlorate sandwiches $[4,5]$. The propellant was made from sheets of APbinder-AP. The AP formed by dry pressing ultra pure AP powder. Four different binders were used. Observations for the combustion were made by high-speed photography and microscopic examination of quenched samples. In addition, Lee et.al. [4] illustrated the effect of inclusion of particulate AP in the binder on the combustion surface and the flame structure. The effect of three types of fuel binder and oxidizer particle diameter on the decomposition and combustion behavior of ammonium perchlorate is studies by $\mathrm{Al}$ Harthi and Williams, [ 6].

Few decades ago, several theoretical studies on the combustion field of the burning of the heterogeneous propellant have been conducted. These researches are divided into two main categories. The first one is concentrated on the gas phase modeling without consideration for the condensed phase process, for example $[7,8,9,10,11]$. The second one is studied the condensed phase reaction as the most important factor, for exampie $[12,13]$.

Recently the complex coupling between the solid-phase and gas-phase process becomes the most factor as a natural step. In particularly, the complexity that arises from the consideration of the unsteady non-planar regression surface. As a result, the coupling of the gas/solid phases, with appropriate jump conditions across the gas/solid interface, it is our belief that it is best followed one small step at a time.

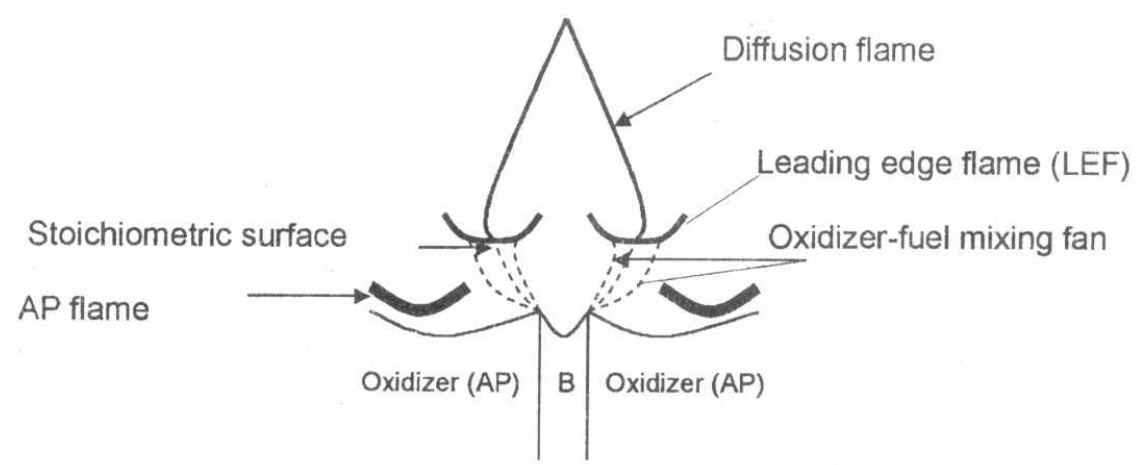

Fig. 2 Flame complex for an AP-Binder-AP sandwich [4]

In the present paper a complete numerical strategy to examine what is perhaps, the simplest model is developed and account the following ingredients: the primary flame between the decomposition products of the binder and the oxidizer (AP), different properties (density, conductivity) of the AP and binder, temperature-dependent gasphase transport properties, an unsteady non-planer regression surface; and a proper accounting of the fluid-mechanics in the gas-phase (retention of the Navier-Stokes equations). These ingredients are applied to the problem of Periodic Sandwich Propellant PSP (alternating slices of AP and binder). 


\section{The PHYSICAL AND MATHEMATICAL MODELS}

In this section the essential component of constant and variable density models is described. The physical model of the PSP is shown in Fig. 3. A model propellant consists of a "sandwich", a sheet of fuel-binder of thickness " $v$ ", layered between two sheets of ammonium perchlorate (AP). Above the solid surface is the gas phase, consisting of a mixture of the decomposition products of the solid oxidizer and fuel. Periodic boundary conditions are applied at $x= \pm L$. The AP-binder-AP sandwich geometry has been recognized as a useful framework in which to gain fundamental insights into propellant combustion (e.g. $[14,15,16])$ and a notable experimental program has been pursued for some years by Price and his colleagues [5]. In addition, combustion behavior of the simpler sandwiches is much easier to observe and describe than combustion of the propellant.

\subsection{Constant and Variable Density Models}

It is useful to summarize the formulation of the constant density model before addressing the complete problem, as this enable us to introduce most of the model ingredients together with various convenient scaling in the context of a model set of equations. The specific details of the constant density model for our problem are as follows: the density is set equal to constant (so that the equation of state, Charles law, is jettisoned); and a uniform velocity field $u=0$ and $v(y)=$ constant is adopted, which satisfies both the continuity and momentum equations. The one-step kinetics that include the primary flame is examined separately in order to achieve a good understanding of the unsteady burning of periodic sandwich propellant with complete coupling between the solid and gas phases. Thus

$$
\text { Fuel }(\mathrm{Y})+\beta \text { Oxidizer }(\mathrm{X}) \stackrel{R}{\Rightarrow} \text { Products }
$$

$R$ is assumed to have the form

$$
R=B\left(P / P_{0}\right)^{n g} \times Y \exp \left(-E_{g} / R_{u} T\right)
$$

Where $B$ is the exponential prefatory, $E_{g}$ is the activation energy in the gas phase, $P$ is the pressure with exponent $n g, R_{u}$ is the universal gas constant, and $(T, X$, and $Y)$ are the temperature, oxidizer, and fuel respectively.

The corresponding gas phase equations are;

$$
\rho_{g} \frac{D \phi}{D t}=\vec{\nabla} \cdot\left(\frac{\lambda_{g}}{c_{p}} \vec{\nabla} \phi\right)+\psi R
$$

where; $\phi=\left[\begin{array}{lll}T & X & Y\end{array}\right]^{T}, \quad \psi=\left[\begin{array}{llll}Q_{g} / c_{p} & -1 & -1 / \beta\end{array}\right]^{T}$ 
When full fluid-mechanics coupling (variable density model) is accounted for, the system of equations ( 3 ) is replaced by;

$$
\frac{\partial Q}{\partial t}+\frac{\partial F}{\partial x}+\frac{\partial G}{\partial y}=H
$$

where;

$$
Q=\left[\begin{array}{l}
\rho_{g} \\
\rho_{g} u \\
\rho_{g} v \\
\rho_{g} e \\
\rho_{g} Y \\
\rho_{g} X
\end{array}\right] ; F=\left[\begin{array}{l}
\rho_{g} u \\
\rho_{g} u^{2}+P-\tau_{x x} \\
\rho_{g} u v-\tau_{x y} \\
\left(\rho_{g} e+P\right) u-\left(u \tau_{x x}+v \tau_{x y}-q_{x}\right) \\
\rho_{g} u Y-\rho_{g} D_{g} Y_{x} \\
\rho_{g} u X-\rho_{g} D_{g} X_{x}
\end{array}\right] ; G=\left[\begin{array}{l}
\rho_{g} v \\
\rho_{g} u v-\tau_{x y} \\
\rho_{g} v^{2}+P-\tau_{y y} \\
\left(\rho_{g} e+P\right) v-\left(u \tau_{x y}+v \tau_{y y}-q_{y}\right) \\
\rho_{g} v Y-\rho_{g} D_{g} Y_{y} \\
\rho_{g} v X-\rho_{g} D_{g} X_{y}
\end{array}\right] ; H=\left[\begin{array}{l}
0 \\
0 \\
0 \\
+Q R \\
-\alpha_{y} R \\
-\alpha_{x} R
\end{array}\right]
$$$$
\text { and } e=\frac{P}{\rho_{g}}+\frac{1}{2}\left(u^{2}+v^{2}\right) ; \tau_{x x}=\mu\left(\frac{4}{3} u_{x}-\frac{2}{3} v_{y}\right) ; \tau_{y y}=\mu\left(\frac{4}{3} v_{y}-\frac{2}{3} u_{x}\right) ; \tau_{x y}=\mu\left(v_{x}+u_{y}\right) \text {; }
$$$$
q_{x}=-\lambda_{g} T_{x} ; q_{y}=-\lambda_{g} T_{y}
$$

Lewis number is taken to be unity, then;

$$
\rho_{g} D_{g}=\lambda_{g} / c_{p}
$$

With the aid of the equation of state;

$$
P=\rho R T
$$

where $\alpha_{x}$ and $\alpha_{y}$ are the stoichiometric coefficients. Here there are six unknowns in the gas-phase, $(u, v, T, X, Y)$ and one unknown in the solid-phase $\left(T_{s}\right)$

\subsection{Solid-Phase and Solid/Gas Interface Equations}

In the solid-phase, the following heat equation is used;

$$
\rho_{s} T_{t}=\frac{\lambda_{s}}{c_{p}} \nabla^{2} T
$$

Here, $\rho_{s}$ is the density of the solid, $T$ the temperature, and $\lambda_{s}$ is the solid thermal conductivity. The specific heat $c_{p}$ is assumed to be equal to that in the gas phase for simplicity. The possibility of differing densities and thermal properties in the solid phase is allowed and setting by; 


$$
\rho_{s}=\left\{\begin{array}{ll}
\rho_{A P} & \lambda_{s}= \begin{cases}\lambda_{A P} & \psi \geq 0 \\
\rho_{B} & \psi<0\end{cases}
\end{array},\right.
$$

The function $\psi(x, y)$ is a level set function which demarks the regions of AP from binder (B) within the solid, so that a point $(x, y)$ lies in the AP if $\psi(x, y) \geq 0$, and in the binder if $\psi(x, y)<0$. Suppose the solid/gas interface defined by $\eta(x(t), y(t), t)=0$. Then;

$$
\eta_{t}+\eta_{x} \frac{d x}{d t}+\eta_{y} \frac{d y}{d t}=0
$$

and the final equation that control the moving of the gas/solid interface as in Fig. 2 is derived by Hegab, et.al. $[14,15]$ and may be written as follows;

$$
\eta_{t}-r_{b}|\vec{\nabla} \eta|=0
$$

where $r_{b}$ is defined as the speed of the front which moves in the directions of the solid. In general $r_{b}$ is a function of $x$ and $t$ and is given by the following simple pyrolysis law;

$$
r_{b}=\left\{\begin{array}{lc}
r_{A P}=A_{A P}\left(P / P_{0}\right)^{n_{A P}} \exp \left\{-E_{A P} / R_{u} T_{A P, s}\right\} & \psi \geq 0 \\
r_{B}=A_{B}\left(P / P_{0}\right)^{n_{B}} \exp \left\{-E_{B} / R_{u} T_{B, s}\right\} & \psi<0
\end{array}\right.
$$

Note that pressure dependence has been added to the pyrolysis law for generality. In the study, the propellant surface is not flat and its shape changes with time. Therefore, the following mapping function is used;

$$
\eta=y-f(x, t)
$$

and the the front of equation (10) reduces to the simple Hamilton-Jacobi equation;

$$
f_{t}+r_{b}(x, t) \sqrt{1+f_{x}^{2}}=0
$$

Further information about the non-planar moving of the gas/solid interfaces using the Level Set strategy is mentioned in details in $[14,15]$.

\subsection{Boundary/Jump Conditions}

The appropriate jump conditions across the gas /solid interface are;

$$
\left[\rho\left(\vec{v} \cdot \vec{n}+r_{b}\right)\right]=0 ;
$$




$$
\begin{aligned}
& {[T]=0 ;} \\
& {[\lambda \vec{n} . \nabla T]=-Q_{s} m ;} \\
& m\left[Y_{i}\right]=\left[\rho D \vec{n} . \nabla Y_{i}\right], i=1,2,3,
\end{aligned}
$$

where $[\phi]=\phi_{\mathrm{g}}-\phi_{\mathbf{s}}$ denotes the jurmp in the quantity $\phi$ across the interface, $m$ is the mass flux. $\vec{n}$ is the unit normal pointing in the direction of the gas; $\vec{n}=\nabla \eta /|\nabla \eta|$, figure (3). $Q_{s}$ is the solid phase heat release term defined by

$$
Q_{s}= \begin{cases}Q_{A P} & \psi \geq 0 \\ Q_{b} & \psi \leq 0\end{cases}
$$

For an exothermic surface reaction, $Q_{s}>0$, and for an endothermic reaction, $Q_{s}<0$. Typically the AP is considered an exothermic reaction, while the binder an endothermic one.

The recent studies by Hegab, et.al. $[14,15]$ proved that the length and time scales for the front and the solid are the same order of magnitudes. On the other side, the ratio of the gas to solid or the ratio of the gas to front are of the order of $10^{-3}$. Thus for the present purpose, the quasi-steady approximation for the gas phase is employed. Note that disturbances with time scales of order $10^{-3} \mathrm{~s}$ would effect the solid phase, but not the gas phase; changes on time scales of order $10^{-5} \mathrm{~s}$ are needed to generate an unsteady gas phase and changes of this nature have been discussed in [11].

\subsection{Nondimensionalization}

The following reference values is taken to nondimensionalize the equations ;

$$
\begin{gathered}
T^{*}=T / T_{\text {ref }}, \quad X^{*}=X / X, \quad Y^{*}=Y / Y_{s}, \quad P^{*}=P / P_{o}, \quad \rho^{*}=\rho / \rho_{o}, \\
\left(u^{*}, v^{*}\right)=(u, v) / V_{g}, \quad f^{*}=f / L, \quad r_{b}^{*}=r_{b} / r_{b, r e f}, \\
\left(x^{*}, \eta^{*}\right)=(x, \eta) / L, \quad t^{*}=t / t_{s}, \quad t_{s}=L / r_{b, r e f}, \quad t_{g}=L / V_{g}, \quad \varepsilon=t_{g} / t_{s} \ll 1 \\
V_{g}=\rho_{A P} r_{b, r e f} / \rho_{g}, \quad T_{\text {ref }}=2700 \mathrm{~K}, Q_{\mathrm{ref}}=\mathrm{c}_{p}{ }^{*} 2700 \mathrm{j} / \mathrm{g}
\end{gathered}
$$

Pressure $p_{0}$ (bar), surface speed $r_{b}$ ref $(c m / s)$, and mass flux $m_{\text {ref }}=\rho_{A P} r_{b}$,ref. Length $L$ (half of the computational domain, which is the sum of the binder and the AP thickness). Time $t=L / r_{b}$,ref. Then the following non-dimensional parameters are 
defined: Peclet numbers $P_{e g}=\rho_{g} V_{g} L c_{p} / \lambda_{g, r e f}$ and $P_{e c}=\rho_{A P} r_{b, r e f} L c_{p} / \lambda_{A P}$, Activation energy $\theta=E /\left(R_{u} T_{\text {ref }}\right)$

$$
\begin{gathered}
\hat{Q}_{s}= \begin{cases}Q_{s, A P} /\left(c_{p} T_{\text {ref }}\right) & \psi \geq 0 \\
Q_{s, B} /\left(c_{p} T_{\text {ref }}\right) & \psi<0,\end{cases} \\
\lambda_{\text {ratio }}= \begin{cases}1 & \psi \geq 0 \\
\lambda_{B} / \lambda_{A P} & \psi<0,\end{cases} \\
\rho_{\text {ratio }}= \begin{cases}1 & \psi \geq 0 \\
\rho_{B} / \rho_{A P} & \psi<0 .\end{cases}
\end{gathered}
$$

In non-dimensional form the equations and boundary/connections form conditions are: $n>0$ for Constant Density Model

$$
\begin{aligned}
& \varepsilon T_{t}+u T_{x}+\bar{v} T_{\eta}=\left(1 / P_{e_{g}}\right) \vec{\nabla} \cdot(\tilde{\lambda} \vec{\nabla} T)+Q_{g} R \\
& \varepsilon X_{1}+u X_{x}+\bar{v} X_{\eta}=\left(1 / P_{e_{x}}\right) \vec{\nabla} \cdot(\tilde{\lambda} \vec{\nabla} X)-R \\
& \varepsilon Y_{1}+u Y_{x}+\bar{v} Y_{\eta}=\left(1 / P_{e_{g}}\right) \vec{\nabla} \cdot(\tilde{\lambda} \vec{\nabla} Y)-R / \beta
\end{aligned}
$$

but temperature-dependent transport is accounted for, viz; $\lambda_{g}=\lambda_{\text {g,ef }} * \tilde{\lambda}\left(T, T_{\text {ref }}\right)$ where $\lambda_{\mathrm{g} \text {,ref }}$ is a reference heat conduction coefficient. The value of $\lambda$ at the reference temperature $T_{\text {ref, }}$ specially $\lambda_{g}=1.08 * 10^{-4} \mathrm{~T}+0.0133$ is choosing with dimensions W/m-K when $T$ is assigned in degrees Kelvin, so that

$$
\begin{aligned}
& \tilde{\lambda}\left(T, T_{\text {ref }}\right)=\frac{1.08 * 10^{-4} T_{\text {ref }} T+0.0133}{1.08 * 10^{-4} T_{\text {ref }}+0.0133} \\
& \mathrm{u}=0, \bar{v}=-\rho_{\text {ratio }} f_{t}, \mathrm{R}=\mathrm{D}_{\mathrm{a}} \mathrm{P} \times \mathrm{Y} \exp \left(-\theta_{\mathrm{g}} / \mathrm{T}\right)
\end{aligned}
$$

\section{$\eta>0$ for Variable Density Model}

The non-dimensional equations of motion can be re-written in the following form:

$\varepsilon \frac{\partial Q}{\partial t}+\frac{\partial F(Q)}{\partial x}+\frac{\partial G(Q)}{\partial y}=\frac{\partial V_{1}\left(Q, Q_{x}\right)}{\partial x}+\frac{\partial V_{2}\left(Q, Q_{y}\right)}{\partial x}+\frac{\partial W_{1}\left(Q, Q_{x}\right)}{\partial y}+\frac{\partial W_{2}\left(Q, Q_{y}\right)}{\partial y}+H$

where, 
$W_{2}\left(Q Q_{y}\right)=\left[\begin{array}{c}0 \\ a u_{x} \\ 4 / 30 w_{x} \\ (\gamma-1) M^{2} \alpha\left\{u u_{y}+4 / 3 v v_{y}\right\}+\beta T_{y} T_{y} \\ \mu / P_{x} X_{y} \\ \mu / P_{e} X_{y}\end{array}\right] V_{2}\left(Q, Q_{y}\right)=\left[\begin{array}{c}0 \\ -2 / 30 w_{y} \\ u_{y} \\ 0 \\ 0\end{array}\right] W_{1}\left(Q_{,} Q_{x}\right)=\left[\begin{array}{c}0 \\ o w_{x} \\ -2 / 30 u_{x} \\ \left.(\gamma-1) M^{2} \alpha_{1}-2 / 3 u v_{y}+v u_{y}\right\} \\ 0 \\ 0\end{array}\right]$

where; $\alpha=\frac{S c}{P_{e}} \mu, \quad \beta^{*}=\frac{S c}{P_{e} P_{r}} \lambda$, and $h=e+\frac{\gamma-1}{\gamma} p / \rho$

\section{$\underline{\eta<0 \text { (Solid Phase) }}$}

$\rho_{\text {ratio }}\left(T_{t}-f_{t} T_{\eta}\right)=\left(\lambda_{\text {ratio }} / P_{e c}\right) \Delta T$

\section{$\underline{\eta=0}$ (Moving Interface)}

$f_{t}+r_{b} \sqrt{1+f_{x}^{2}}=0$,

$T\left(x, 0^{+}, t\right)=T\left(x, 0^{-}, t\right)$

$\left.\hat{\lambda}\left(\frac{-f_{x} T_{x}+\left(1+f_{x}^{2}\right) T_{\eta}}{\sqrt{\left(1+f_{x}^{2}\right)}}\right)\right|_{0^{\circ}}-\left.\hat{\lambda} \lambda_{\text {rato }}\left(\frac{-f_{x} T_{x}+\left(1+f_{x}^{2}\right) T_{\eta}}{\sqrt{\left(1+f_{x}^{2}\right)}}\right)\right|_{0^{-}}=-\hat{Q}_{x} m P_{e_{x}}$

$\left.\left(m X-\frac{\hat{\lambda}}{P_{\boldsymbol{o}_{s}}}\left\{\frac{-f_{x} X_{x}+\left(1+f_{x}^{2}\right) X_{\eta}}{\sqrt{\left(1+f_{x}^{2}\right)}}\right\}\right)\right|_{0^{*}}=\left\{\begin{array}{cc}m & \psi \geq 0 \\ 0 & \psi<0\end{array}\right.$

$\left.\left(m Y-\frac{\hat{\lambda}}{P_{e_{g}}}\left\{\frac{-f_{x} Y_{x}+\left(1+f_{x}^{2}\right) Y_{\eta}}{\sqrt{\left(1+f_{x}^{2}\right)}}\right\}\right)\right|_{0^{+}}= \begin{cases}0 & \psi \geq 0 \\ m & \psi<0\end{cases}$

$\underline{\eta \rightarrow \infty} \quad \mathrm{T}=\mathrm{T}$ 。 
$\underline{\eta \rightarrow+\infty} \quad \frac{\partial}{\partial \eta}(\cdot)=0$

$|x|=1 \quad$ Periodic boundary conditions

\section{NUMERICAL METHODS}

\subsection{Modified Conservation Equations}

To avoid pressure singularity at low Mach numbers, the numerical strategy outlines in [18] is used. The pressure is rescaled in the momentum equation since it is the pressure gradient, not the actual pressure, that is involved in the momentum balance. The rescaled pressure is applied to retain accuracy in calculating the momentum conservation. As a result, the pressure is divided into constant and fluctuating parts as follow;

$$
P(x, y, t)=1+\gamma M^{2} \hat{P}(x, y, t)
$$

and substitute into the equations of motion given above. Where $M$ is the Mach number $\left(M=V / C_{0}\right)$. The equation of state now becomes;

$$
\rho=\frac{1+\gamma M^{2} \hat{P}(x, y, t)}{T}
$$

and is used to update the density. Since the quasi-steady state solution in the gas phase $(\varepsilon<<1)$ is required, the physical time term in $(25)$ is eliminated. In addition an accelerate convergence technique is employed [18]. The technique begins by adding a pseudotime derivative to the conservation equations (25). So the equations to be solved for the gas phase become;

$\Gamma \frac{\partial \tilde{Q}}{\partial \tau}+\frac{\partial F(Q)}{\partial x}+\frac{\partial G(Q)}{\partial y}=\frac{\partial V_{1}\left(Q, Q_{x}\right)}{\partial x}+\frac{\partial V_{2}\left(Q, Q_{y}\right)}{\partial x}+\frac{\partial W_{1}\left(Q, Q_{x}\right)}{\partial y}+\frac{\partial W_{2}\left(Q, Q_{y}\right)}{\partial y}+H$

where $\tau$ represents the pseudo-time domain. Because the pseudo-time derivative vanishes at convergence to the steady state solution in the gas phase, a certain amount of liberty is given in choosing the variables in $\tilde{Q}$. A new scaled pressure term $\tilde{P} / \beta$ is added as a pseudo-time derivative ternil to the continuity equation. The remaining variables in $\tilde{Q}$ are then fixed by rewriting the momentum, energy, and species equations from their nonconservative form by means of the modified equation of mass conservation. As a result, the pseudo-time variable vector $\tilde{Q}$ and its associated preconditioning matrix $\Gamma$ takes the form; 


$$
\tilde{Q}=\left[\begin{array}{l}
\hat{P} \\
u \\
v \\
h \\
Y \\
X
\end{array}\right] \Gamma=\left[\begin{array}{ccccccc}
1 / \beta^{\prime} & 0 & 0 & 0 & 0 & 0 \\
u / \beta^{\prime} & \rho & 0 & 0 & 0 & 0 \\
v / \beta^{\prime} & 0 & \rho & 0 & 0 & 0 \\
h / \beta^{\prime}-1 & 0 & 0 & \rho & 0 & 0 \\
Y / \beta^{\prime} & 0 & 0 & 0 & \rho & 0 \\
X / \beta^{\prime} & 0 & 0 & 0 & 0 & \rho
\end{array}\right]
$$

Factor $\beta^{\prime}$ is used to properly scale the time derivative of $\hat{p}$. Its selection is proportional to the dynamics pressure of the flow field.

\subsection{ADI Algorithm Development}

The first step for advancing the solution of (37) is using the Delta form scheme [19] as follows ;

$$
\Delta Q^{n}=\frac{\theta \Delta \tau}{1+\xi} \frac{\partial}{\partial \tau}\left(\Delta Q^{n}\right)+\frac{\Delta \tau}{1+\xi} \frac{\partial}{\partial \tau}\left(Q^{n}\right)+\frac{\xi}{1+\xi} \Delta Q^{n-1}+O\left[(\theta-1 / 2-\xi) \Delta \tau^{2}+\Delta \tau^{3}\right]
$$

and $\Delta Q^{n}=Q^{n+1}-Q^{n}$

The time-difference formulas (39) and (40), with the appropriate choice of the parameters $\xi$ and $\theta$ reproduce many familiar two and three level, implicit schemes. The three level, second order accuracy implicit schemes $(\xi=1 / 2$ and $\theta=1)$ with special treatment for the cross derivative terms at the level $(n-1)$ is applied. By inserting the temporal derivative of equation (37) in (39) and by straightforward derivation, the resulting approximated form can be splitted into two-tridiagonal systems:

$\left[I+\theta \Delta \tau \Gamma^{-1}\left(\frac{\partial}{\partial x}\left(A-P+R_{x}\right)^{n}-\frac{\partial^{2}}{\partial x^{2}}\left(R^{n}\right)\right)\right] \Delta Q^{*}=\Gamma^{-1} R H S$

$\left[I+\theta \Delta \tau \Gamma^{-1}\left(\frac{\partial}{\partial y}\left(B-\tilde{Q}+S_{y}\right)^{n}-\frac{\partial^{2}}{\partial y^{2}}\left(S^{n}\right)\right)\right] \Delta Q=\Delta Q^{*}$

where $A^{n}, P^{n}, R^{n}, R^{n}{ }_{x} B^{n}, \tilde{Q}^{n}, S^{n}$, and $R^{n}$ are the jacobian matrices for the implicit $\mathrm{x}$ - and y-sweep $(41,42)$ respectively, These matrices are derived and written in Appendix $(A)$. $I$ is a unit matrix (6X6) and $\Gamma^{-1}$ is the inverse of the preconditioning matrix.

The three-points second order accuracy central difference approximation are used for the spatial differences in the LHS of x-sweep form (41). This approximation along with 
the applied periodic boundary conditions at $|x|=1$ produce a system of Periodic BlockTridiagonal Equations (PBTE). After the computation of $\Delta Q^{*}$ at the interior points by solving this system of PBTE with each block having dimensions $6 \times 6$ components, the code is ready for the implicit y-sweep form (42). Here again, the three-points second order accuracy central difference approximation are used for the spatial differences in the LHS of (42). This approximation along with the rigid wall boundary conditions at $y=0$ produce a system of Non-Periodic Block-Tridiagonal Equations (NPBTE). The final delta form $\triangle Q$ can be computed by solving the NPBTE system. Then the solution at new time step $(n+1)$ can be determined from Eqn.(41). Note that, the cross-derivatives terms at $(n-1)$ are treated explicitly to avoid the implicit coupling of adjacent boundary points.

\subsection{Numerical Solution to Hamilton-Jacobi Equation}

The surface equation (13) is solved in order to follow the non-planar regression surface by the first order temporal scheme [20];

$$
f_{i}^{n+1}=f_{i}^{n}-\Delta t \phi\left(\frac{f_{i}^{n}-f_{i-1}^{n}}{\Delta x}, \frac{f_{i+1}^{n}-f_{i}^{n}}{\Delta x}\right)
$$

where $\phi$ is a numerical flux function. Here, $i$ denotes the discrete grid location $x_{i}, n$ the previous time level, and $n+1$ the new time level. Although there are many choices, the second-order Lax-Freidricks monotonic flux function is used;

$$
\phi_{L F}\left(u_{1}, u_{2}\right)=\frac{1}{2}\left(\frac{r_{b, i}+r_{b, i-1}}{2} \sqrt{1+u_{1}^{2}}+\frac{r_{b, i+1}+r_{b, i}}{2} \sqrt{1+u_{2}^{2}}\right)
$$

and $r_{b, l}$ is the local burn rate determined from the pyrolysis law. The CFL. condition $\Delta t \leq C F L \Delta x$ is satisfied for stability. Typically one would set $C F L=\left(\max \left\{r_{b}\right\}\right)^{-1}$; however the value of $1 / 4$ seems satisfactory.

Beside the non-flat regression surface mapping as in (11), another transformation is applied for the cluster grid points in regions adjacent to the wall, where most of the flow parameters changes rapidly. The solution of the final mapped equations is advanced in the solid phase using physical time $(t)$. Simultaneously the solution in the gas phase using pseudo-time $(\tau)$ to the local steady state at the first physical time step (t) is advanced. The boundary/jump conditions are continually updated as in Eqs. (26) to (34). Then the Hamilton-Jacobi Eqn. (13) is advanced at the physical time by a third order ENO and a fifth-order WENO (weighted essentially non-oscillatory) solver $[21,22]$. All numerical calculations were performed on a $140 \times 70$ grid, uniform in the x-direction and stretched in the $y$-direction. The code in the gas phase is stopped between each two physical times when the relative difference between solutions at two different pseudo-time values is less than some prescribed tolerance, taken here to be $10^{-6}$. Convergence tests where carried out and it was determined that any further refinement resulted in less than $1 \%$ relative error. 


\section{4) RESULTS AND DISCUSIONS}

The understanding of the complex combustion structure of AP-Binder-AP sandwich, as a simple model to the heterogeneous solid rocket propellant, is studied in details by two different models. The first model is the constant density model and the second one is the variable density model or the Navier-Stokes model. Initially, the solution starts from a flat surface $f(x, t=0)=0$. Then the solution is advanced in the solid phase, gas phase and the moving simultaneously as it is mentioned above. The first set of the results are for the constant density model. In this model, it has been taken $\mu=\Lambda\left(T, T_{\text {ref }}\right)$ which is one of the more realistic choices rather $\mu=$ constant in our earlier studies [7]. In the gas phase, the contours plots for the fuel-vapor $(Y)$, oxidizer $(X)$, gas temperature $\left(T_{g}\right)$, and reaction rate contours $(R)$ are shown in figure (5) for for $\theta=7, \beta=7, P_{e}=6, D_{a g}=5^{*} 10^{6}, P=10$. The upper portion represent the gas phase and the lower one refer to the solid phase. The dark gray region in the latter represent the binder layers between the two AP sheets (light gray). The contour values are written in the upper part of each plot. The fuel vapor is generating from the binder and diffuses to a certain distance in the far-field. The maximum contour value is $Y_{\max } \sim 0.65$, representing the inner small arc near to the surface, while the outer contour show the smallest value $Y_{\min } \sim 0.05$. Similarly, the oxidizer concentration $(X)$ diffuses over the AP sheets in the same manner. In contrast, the temperature contours attains the smallest values near the burning surface $\left(\mathrm{T}_{\mathrm{w}} \sim 0.4^{\star} 2700 \sim 1080 \mathrm{~K}\right)$ and the largest values representing the flame temperature cover the far-field in the gas phase $\left(T_{r} \sim 0.95^{\star} 2700 \sim 2565 \mathrm{~K}\right)$ nearly at $y>1.5$. The last plot of figure (5) show the location and shape of the generating flame. These reaction rate contours show that the flame is characterized by two strong mixing structure each centered at $|x| \sim 0.223$ with maximum values $R R_{\max } \sim 2.5$, representing the inner cell on each side, while the outer contours exhibit the smallest values $R R_{\min } \sim 0.25$.

Figure (6) shows the reaction rate contours at several times. The successive curved shapes through the solid phase show that, the surface is initially flat and then as the solution is advanced, the combustion surface retreats in an unsteady fashion and finally retreats at a fixed speed with unchanged shape by $t=2.49$. These contours reveal not only the significant effect of the surface profiles and the burning rate on the shape of the flame but also on the maximum reaction rate values which decrease as time increase. The differences in profiles with time reflect the behaviour of the burning rate at the propellant surface and reconstruct the corresponding flame structures and identify the parts of the flame structure that dominate the sandwich burning rate and the surface heat flux. In addition there is a sharp discontinuity in slope at the interface between the binder and AP regions. This interesting phenomena is predicted in [23].

Figure (7) show a comparison between the experimental image (the left) [24] and the current computation model (the right) for the structure of the flame shape and the burning surface. The stoichiometric level surface show the location at which the fuel and oxidizer meet together in shoichiometric proportions. The inner region for the stoichiometry contour is the fuel-rich region (see the fuel vapour image), while the area 
out of the stoichiometry envelop represent the fuel-lean composition. The two stoichiometric surface in both the computational and experimental images represent the two strong mixing structure (at the AP-Binder interface) each centered at $|x| \sim 0.223$ and forming the flame envelop. This comparison show how the computational steadily surface regressing profile (at $t>5$ ) is qualitatively consistent with the experimental emission-transmission composite image by Brewster, et al. (2001), [24] and also with the experimental photography for the spontaneous quench samples of AP/BPAN/AP sandwich that reported by Lee et al. [4]. Both theoretical and experimental results verified that conditions that gave the highest burning rates resulted in narrow smooth bands of binder in the middle with little "protrusion" of AP at the interface.

The effect of the exposed pressure on the burning surface profiles and the flame positions and shapes are presented in the second group of the first set for the constant density model(1). Figure (8) show the reaction rate contours at three pressure values $P=1,10,15$ in the gas phase at $t=0.06249$ for $\theta=7, \beta=7, P_{e}=6, D_{a g}=5^{*} 10^{6}$. This figure show not only the effect of the pressure on the flame shape but also the significant effect on the maximum reaction rate contours, where $R R_{\max } \leq 1$ for $P=1, R R_{\max } \leq 2.5$ for $P=10$, and $R R_{\max } \leq 3.6$ for $P=15$. In addition the flame is moving or setting closer and closer to the combustion surface as the pressure increases. Furthermore, the effect of the pressure on the surface heat flux;

$$
\frac{\partial T}{\partial n}=n \cdot \nabla T=\frac{-f_{x} \frac{\partial T}{\partial \xi}+\left(1+f_{x}^{2}\right) \frac{\partial T}{\partial \eta}}{\sqrt{1+f_{x}^{2}}}
$$

is presented in figure(9). This result reveal that the heat flow vector, near the gas-solid interface and AP-binder solid interface, is from the binder toward the AP in the solid phase. Consequently there must exist a weak (hot) portion of the binder slightly up from the corner interface and into the binder. This might account for the appearance of the Vshape (notches) in the binder. This phenomena have been seen in the experimental quenched samples by Handley, et al. [23].

A comparison between the experimental image and the computational model for the response of the combustion surface profiles to the pressure change (experimental at 7 $\mathrm{atm}$ in the left and the computational at 1,10, and $15 \mathrm{~atm}$ in the right) is presented in figure(10). Both images illustrate that increasing the pressure causes the binder to stick out and the surface consist of a "trough" centered on the binder lamina. In addition a comparison of these clear pictures with the experimental results by Price and et al. [5] is possible but caution is appropriate as the sandwiches that Price examined are isolated, not periodic.

The last set of the results are for the variable density model(2). In this section the results obtained from the constant density model(1) with those obtained using the NavierStokes equations, model(2) are compared, and thus to validate the simpler strategy. Figure(11) shows a comparison between the two models for the fuel-vapor (a) 
and reaction rate contours (b) for $\theta=7, \beta=7, P_{e}=6, D_{a g}=5^{\star} 10^{6}$ and $P=10$ at $t=0.06429$. It is noted that the flame is setting at the same transverse location with slight differences in the maximum reaction rate contours. $R R_{\max }$ is 2.88678 for model(2), compared to 2.66972 for model(1) and in the adiabatic flame temperature, where $T^{\prime}$ max is $2448.09 \mathrm{~K}$ for model(2) compared to 2446.57 for model(1). Figure(11-b) shows that there is only a small difference in the far-field for the fuel-vapor contours $(Y)$ for the two models. These very slight differences are related to the slow flow accelerated since the axial velocity component $u$ is not zero when the Navier-Stokes equations are used and the transverse velocity component becomes more or less in the neighborhood of the flame for model(2). Figure(12) shows the axial velocity contours at different times for the NavierStokes model. It is noted that an axial velocity is generated near the combustion surface due to the surface morphology, where the velocity cells are positive on the left hand side, since the flow goes down hill and the right hand side cells are negative, since the flow goes up hill. Moreover, the absolute value of the axial velocity increases as time increases, since the curvature in the combustion surface profiles become more deeper than at earlier time.

The small differences between the two models reveal that, a useful exploration calculations can be carried out using the constant density model, since the generated axial velocity component is very small, $0.0 \leq|u| \geq 0.04$ and also the variation in $v$ is consistent with the mass conservation. In general, the Navier-Stokes model, it is our believe, may have a significant effect when the current solution to the modeling of randomly packed heterogeneous propellant, particularly in 3-D solution is advanced.

\section{CONCLUSION}

Few decades ago the details of the heterogeneous propellant combustion has been discussed. The theoretical framework has consists of a $2 \mathrm{D}$ physical pictures, but most modeling efforts have been 1D mathematical model as in [1,2,3, and 4]. This has obviously been of value and important insights have been achieved, but the to adopt a multidimensional numerical framework is required.

Here, for the first time, the $2 \mathrm{D}$ calculations to the combustion of heterogeneous solid propellant, accounting for the gas phase physics, the solid phase physics and an unsteady non-planar description of the regressing propellant surface is developed. There are a number of issues that have discussed. The speed within which the combustion surface recedes depends on the exposed pressure in the gas phase, the effect of several parameters on the combustion and shape of the flame. In addition a variety of steady-state surface shapes are achieved. At higher pressure values, the binder is tend to stick out and the surface consists of a "trough" centered on the binder lamina. These trends were also recognized in an experimental observation [5].

A comparison between the computational steadily surface regressing profile with the experimental emission-transmission composite image by Brewster, et al. (2001), [24] 
and also with the experimental photography for the spontaneous quench samples of AP/BPAN/AP sandwich that reported by Lee et al. [4] is qualitatively consistent.

In this study, the Navier-Stokes equations were used rather the constant density model in earlier work $[7,10,14$, and 16]. A comparison between the constant density model and the Navier-Stokes solutions reveals very small differences. As a results, the NavierStokes model may have a significant effect when our solution to the modeling of randomly packed heterogeneous propellant, particularly in 3-D solution is advanced.

\section{Acknowledgments}

This work was supported by the U.S. Department of Energy through the University of California under Subcontract number B341494. A useful discussion with the research team at the Center for Simulation of Advanced Rocket (CSAR), University of Illinois at Urbana-Champaign (UIUC), USA is appreciated.

\section{References}

1. M.W. Beckstead, R.L. Derr, and C.F. Price, "A Model of Composite SolidPropellant Combustion Based on Multiple Flames", AIAA Journal, 8(12):22002207, (1970).

2. B. Rasmussen and Jr. R.A. Frederick, "A Nonlinear Heterogeneous Model of Composite Solid Propellant Combustion", AIAA Paper No. 99-2228, In $35^{\text {th }}$ AIAAIASME/SAE/ASEE Joint Propulsion Conference, June (1999).

3. J.J. Murphy and H. Krier , "Heterogeneous effects on Dynamic Burning in Composite Solid Propellant" Proceeding of the Combustion Institute, 28, (2000).

4. S. Lee, E. Price, and R. Sigman, "Effect of Multidimensional Flamelets in Composite Propellant Combustion", ", Journal of Propulsion and Power, Vol.10(6), PP 761-768, (1994).

5. E.Price, J.Handley, R.Panyam, R.Sigman, and A.Ghosh, "Combustion of Ammonium Pechlorate-Polymer Sandwiches", AIAA Journal, Vol.19, No. 3, PP, 380-386, (1981).

6. A. Al-Harthi and A. Williams, "Effect of fuel binder and oxidizer particle diameter on the combustion of ammonium perchlorate based propellants" Fuel, Vol. 77, No. 13, pp1451-1468, (1998).

7. T.L.Jackson, J. Buckmaster, and A. Hegab, "Periodic Propellant Flames and Fluid-Mechanical Effects", Vol. 17, Number 2, Pages 371-379, (2001).

8. J. Buckmaster, T.L. Jackson, and J. Yao, "An Elementary Diffusion of Propellant Flame Geometery", Combustion and Flame Vol. 117, PP 541-552, (1999).

9. T.L.Jackson, J. Buckmaster, and J. Hoeflinger, "Three-Dimensional Flames Supported by Heterogeneous Propellants", JANNAF Paper, JANNAF CSIPSHSIAPS Joint Meeting, Cocoa Beach, FL. October (1999). 
10. T.L. Jackson and J. Buckmaster, "Nonpremixed Periodic Flames Supported by Heterogeneous Propellant", Journal of Propulsion and Power, Vol.16(3), PP 498504, (2000).

11. W. Cai and V. Yang, "A Model of AP/HTPB Composite Propellant Combustion", AIAA Paper 2000-0311, 38 $8^{\text {th }}$ Aerospace Science Meeting (2000).

12. R.H. Waesche and J. Wenograd, "Calculation of Solid Propellant Burning Rate from Condensed-Phase Decomposition Kinetics", AIAA Paper 69-145, N. Y., Jan. (1969).

13. C.E. Hermance, "A Model of Composite Propellant Combustion Including Surface Heterogeneity and Heat Generation", AIAA Journal, Vol.4 PP 1629-1637, (1960).

14. A. Hegab, T. Jackson, J. Buckmaster, and S. Stewart, "The Burning of Periodic Sandwich Propellants", $36^{\text {th }}$ AIAA/ASME/SAE/ASEE Joint Propulsion Conference, AIAA Paper 2000-3459, (2000).

15. A. Hegab, T. Jackson, J. Buckmaster, and S. Stewart, "Nonsteady Burning of Periodic Sandwich Propellant with Complete Coupling between the Solid and Gas Phases" Combustion and Flame, Vol. 125(1/2), PP 1055-1070, (2001).

16. G.M. Knott and M.Q. Brewster, "Two-Dimensional Combustion Modeling of Heterogeneous Propellants with finite Peclet Number", Combustion and Flame, Vol. 121(1/2), PP 91-106, (2000)

17. I.S. Tseng and V. Yang, "Combustion of a Double-Base Homogeneous Propellant in a Rocket Motor", Combustion and Flame 96:325-342, (1994).

18. T.S. Roh and V. Yang, "Transient Combustion Responses of Solid Propellants to Acoustics Disturbances in Rocket Motors", AIAA Paper 95-0602, (1995).

19. R.M. Beam and R.F. Warming, "An Implicit Factored Scheme for the Compressible Navier-Stokes Equations" AIAA Journal, Vol.16, No.4, April, 1978.

20. M.K. King, "Examination of Chemical Approaches to stabilizing Composite Propellant Combustion", Journal Propulsion and Power, Vol. 12, PP 554-563, 1996.

21. A. Harten, "Uniformly High Order Accurate Essentially Non-oscillatory Schemes, III", Journal of Computational Physics, 71, 231-303, (1987).

22. G. Jiang and C. Shu "Efficient Implementation of Weighted ENO Scheme", Journal of Computational Physics, 126, 202-228, (1996).

23. Handley, J.C. and Strahle, W.C. "The Behaviour of Several Catalysts in the Combustion of Solid Propellant Sandwiches", AIAA Paper 74-122, Washington, D.C., (1974)

24. M.Q. Brewster, G.M. Knott, B.T. Chorpening, "Combustion of AP/HTPB Laminate Propellants”, AIAA Paper 2001-4501, 37 ${ }^{\text {th }}$ AIAA/ASME/SAE/ASEE Joint Propulsion Conference, Salt Lake City, UT, USA, (2001). 
$\operatorname{Appendix}(\mathrm{A})$ :

The Jacobian Matrices of Eqns. (41) and (42) are derived as;

$$
\begin{aligned}
& A^{n}=\left(\frac{\partial F}{\partial \tilde{Q}}\right)^{n}=\left[\begin{array}{cccccc}
0 & \rho & 0 & 0 & 0 & 0 \\
1 & 2 \rho u & 0 & 0 & 0 & 0 \\
0 & \rho v & \rho u & 0 & 0 & 0 \\
0 & \rho h & 0 & \rho u & 0 & 0 \\
0 & \rho Y & 0 & 0 & \rho u & 0 \\
0 & \rho X & 0 & 0 & 0 & \rho u
\end{array}\right] \quad R^{n}=\left(\frac{\partial V_{1}}{\partial \tilde{Q}_{-}}\right)^{n}=\left[\begin{array}{cccccccc}
0 & 0 & 0 & 0 & 0 & 0 & \\
0 & 4 / 3 \alpha & 0 & 0 & 0 & 0 & \\
0 & \alpha & 0 & 0 & 0 \\
0 & 0 & & & & \\
0 & (\gamma-1) M^{2}\left(4 / 3 \alpha-\beta^{*}\right) u & (\gamma-1) M^{2}\left(\alpha-\beta^{*}\right) \nu & \beta^{*} & 0 & 0 \\
0 & 0 & & 0 & 0 & \mu / P_{e} & 0 \\
0 & 0 & & 0 & 0 & 0 & \mu / P_{e}
\end{array}\right] \\
& B^{n}=\left(\frac{\partial G}{\partial \tilde{Q}}\right)^{n}=\left[\begin{array}{cccccc}
0 & 0 & \rho & 0 & 0 & 0 \\
0 & \rho v & \rho u & 0 & 0 & 0 \\
1 & 0 & 2 \rho v & 0 & 0 & 0 \\
0 & 0 & \rho h & \rho \nu & 0 & 0 \\
0 & 0 & \rho Y & 0 & \rho v & 0 \\
0 & 0 & \rho X & 0 & 0 & \rho \nu
\end{array}\right] \quad S^{n}=\left(\frac{\partial W_{2}}{\partial \tilde{Q}_{y}}\right)=\left[\begin{array}{cccccccc}
0 & 0 & 0 & 0 & 0 & 0 & \\
0 & \alpha & 0 & 0 & 0 & 0 & & \\
0 & 0 & 4 / 3 \alpha & 0 & 0 & 0 & & \\
0 & (\gamma-1) M^{2}\left(\alpha-\beta^{*}\right) u & (\gamma-1) M^{2}\left(4 / 3 \alpha-\beta^{*}\right) \nu & \beta^{*} & 0 & 0 \\
0 & 0 & 0 & 0 & \mu / P_{e} & 0 \\
0 & 0 & & 0 & 0 & 0 & \mu / P_{t}
\end{array}\right]
\end{aligned}
$$

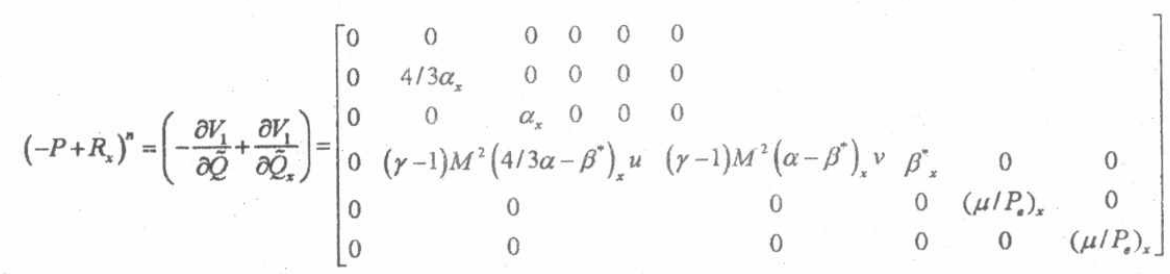

$$
\begin{aligned}
& \left(-Q+S_{y}\right)^{n}=\left(-\frac{\partial W_{2}}{\partial \bar{Q}}+\frac{\partial W_{2}}{\partial \tilde{Q}_{y}}\right)=\left[\begin{array}{ccccccccc}
0 & 0 & 0 & 0 & 0 & 0 & & & \\
0 & \alpha & 0 & 0 & 0 & 0 & & & \\
0 & 0 & 4 / 3 \alpha_{y} & 0 & 0 & 0 & & & \\
0 & (\gamma-1) M^{2}\left(\alpha-\beta^{*}\right)_{y} u & (\gamma-1) M^{2}\left(4 / 3 \alpha-\beta^{*}\right)_{y}{ }^{\nu} & \beta^{*} y_{y} & 0 & 0 \\
0 & 0 & 0 & 0 & 0 & \left(\mu / P_{e}\right)_{y} & 0 \\
0 & 0 & & 0 & 0 & 0 & \left(\mu / P_{e}\right)_{y}
\end{array}\right]
\end{aligned}
$$

and the Right Hand Side (RHS) of Eq. (41) is derived as:

$$
R H S=\Gamma^{-1}\left\{\begin{array}{l}
\left(-\frac{\partial F(Q)}{\partial x}-\frac{\partial G(Q)}{\partial y}+\frac{\partial V_{1}\left(Q, Q_{x}\right)}{\partial x}+(1+\hat{\theta})\left[\frac{\partial V_{2}\left(Q, Q_{y}\right)}{\partial x}+\frac{\partial W_{1}\left(Q, Q_{x}\right)}{\partial y}\right]+\frac{\partial W_{2}\left(Q, Q_{y}\right)}{\partial y}+H\right)^{n} \\
-\theta\left[\frac{\partial V_{2}\left(Q, Q_{y}\right)}{\partial x}+\frac{\partial W_{1}\left(Q, Q_{x}\right)}{\partial y}\right]^{n-1}
\end{array}\right\}
$$

where $\Gamma^{-1}$ is the inverse of the preconditioning matrix in Eqn. (38). 
Table 1: Thermophysical properties of the gas, AP, and Binder are fitted to the experimental data. References to the values chosen in this study have been cited. The values of $A_{A P}$ and $A_{B}$ have been Chosen so that the pyrolysis law yields $r_{b}$,ref $=0.25 \mathrm{~cm} / \mathrm{s}$ at $860 \mathrm{~K}$. Most of the gas phase properties have been made up to approximately match our old results without solid coupling [4].

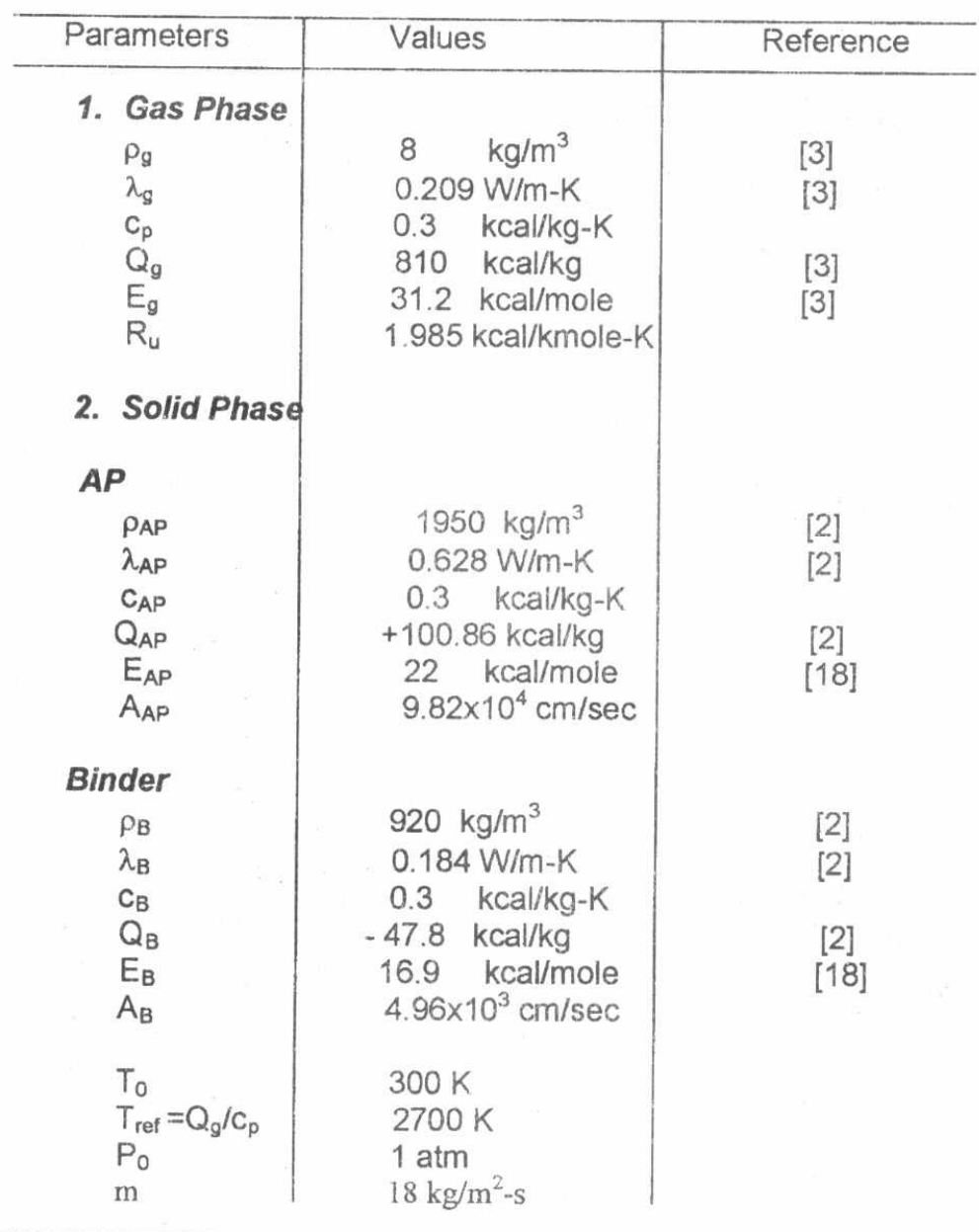




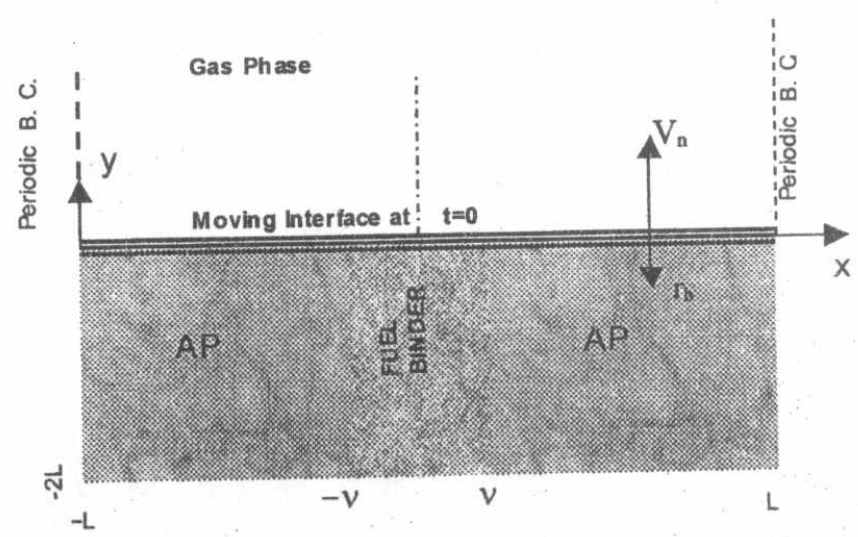

Fig. 3. Periodic sandwich propellant configuration

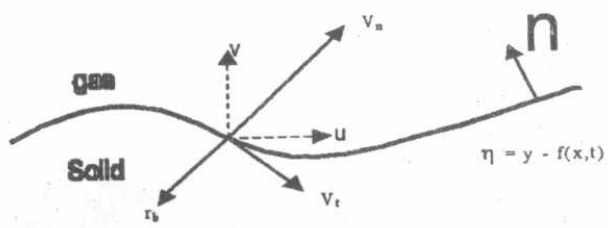

Fig. 4. Sketch showing the coordinate system for the moving surface

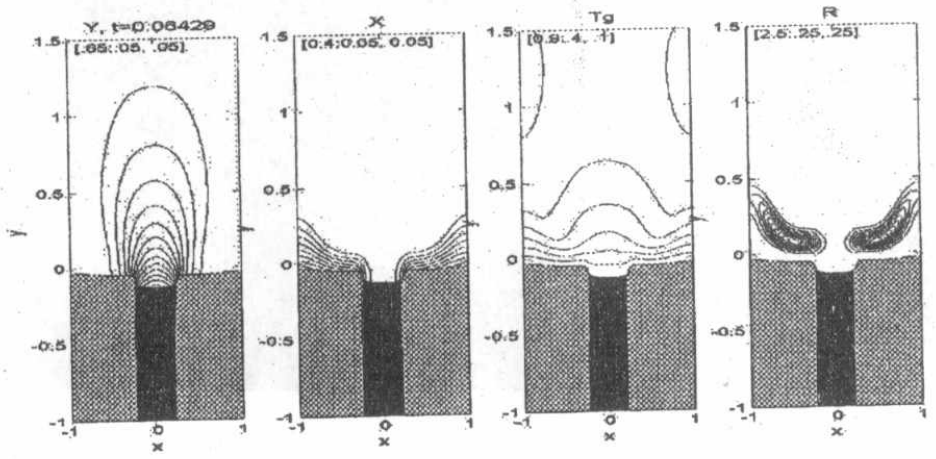

Fig. 5. Fuel $\operatorname{vapor}(Y)$, oxidizer $(X)$, temperature $(T g)$, and reaction rate $(R)$ contours in the gas at time $t=0.06249$. 


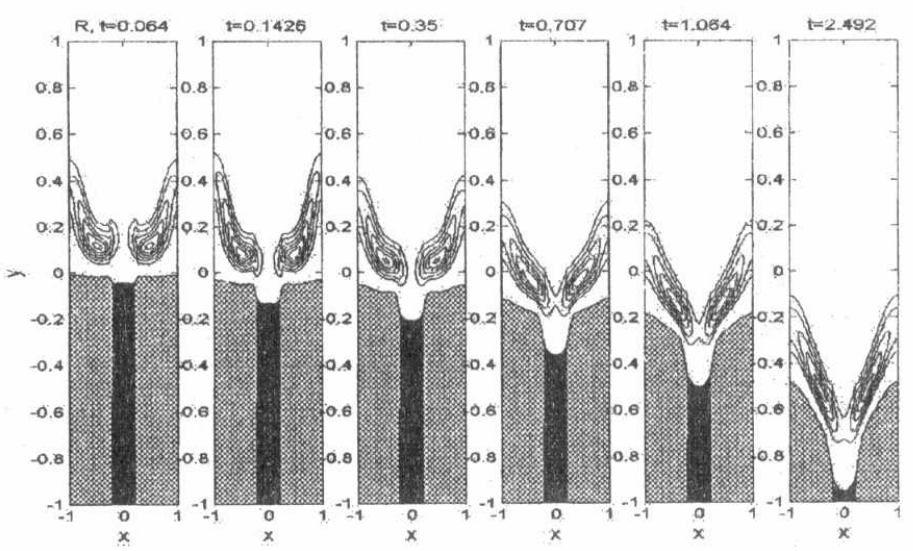

Fig. 6. Reaction rate contours at different times in the gas-phase for the flow parameters as in Fig. 5.

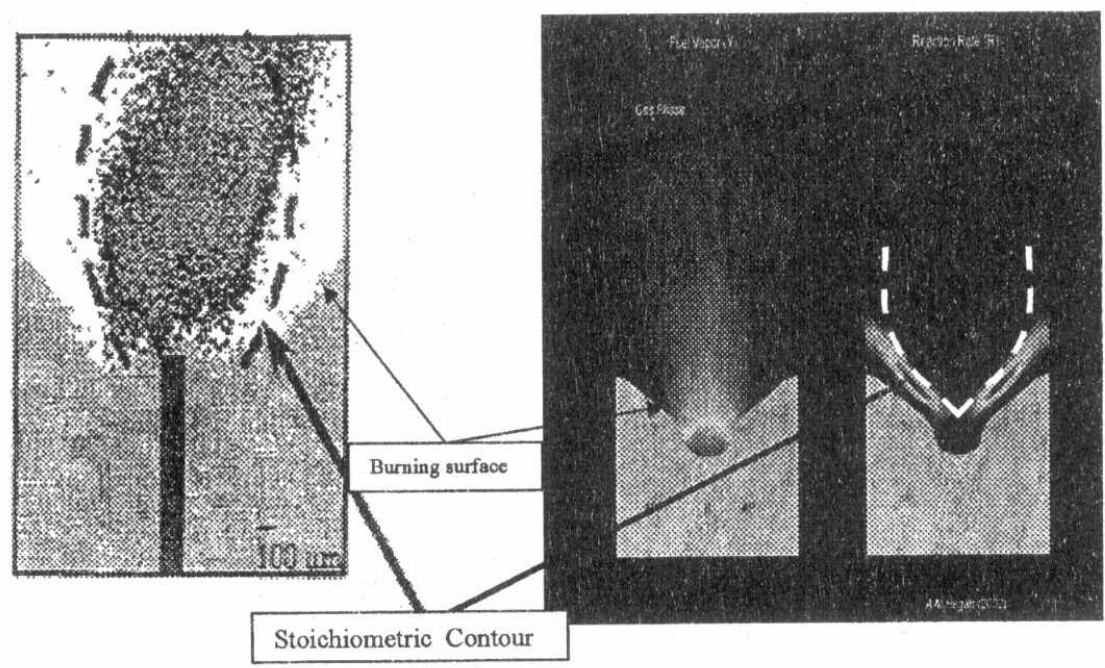

Fig. 7. Comparison of experimental image for the burning of AP-Binder-AP sandwich[24] \{left\} with the numerical model \{right\}. 

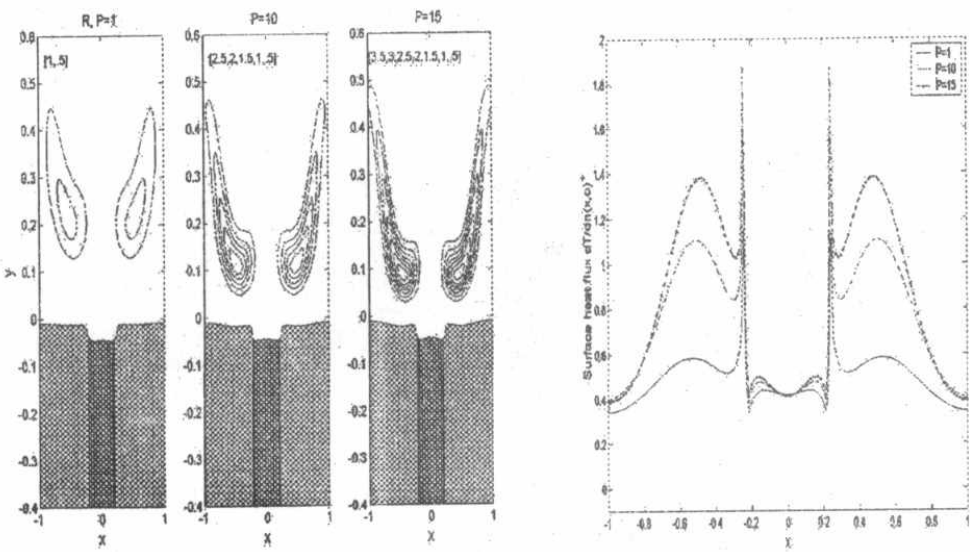

Fig. 8. Effect of pressure on flame surface structure.
Fig. 9. Effect of pressure on the surface heat flux $d T / d n(x, 0)$

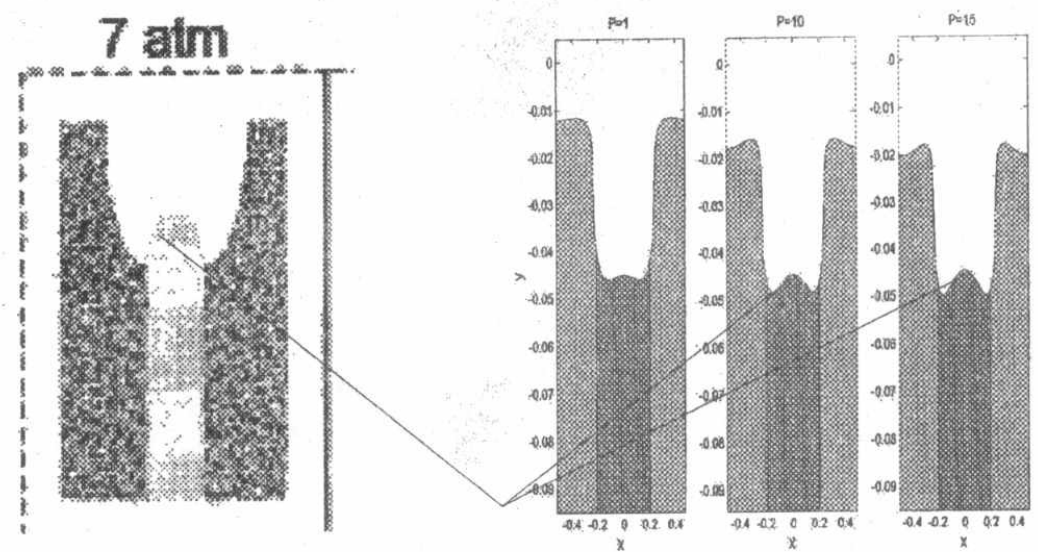

Fig. 10. Effect of pressure on the burning surface structure. Left figure is experimental [5] and the right figures are the computational model. 

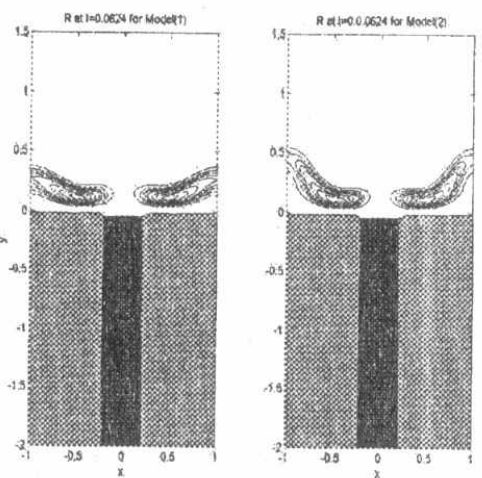

(a)
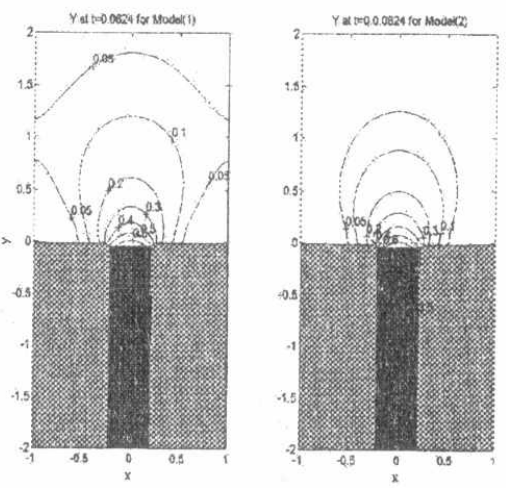

(b)

Fig. 11. Comparison of reaction rates (a) and fuel vapour (b) for the constant density model (1) and the variable density model(2) for $\theta=7, \beta=7, P_{e}=6$, $D_{a g}=5^{\star} 10^{6}, P=10, M=0.02$.
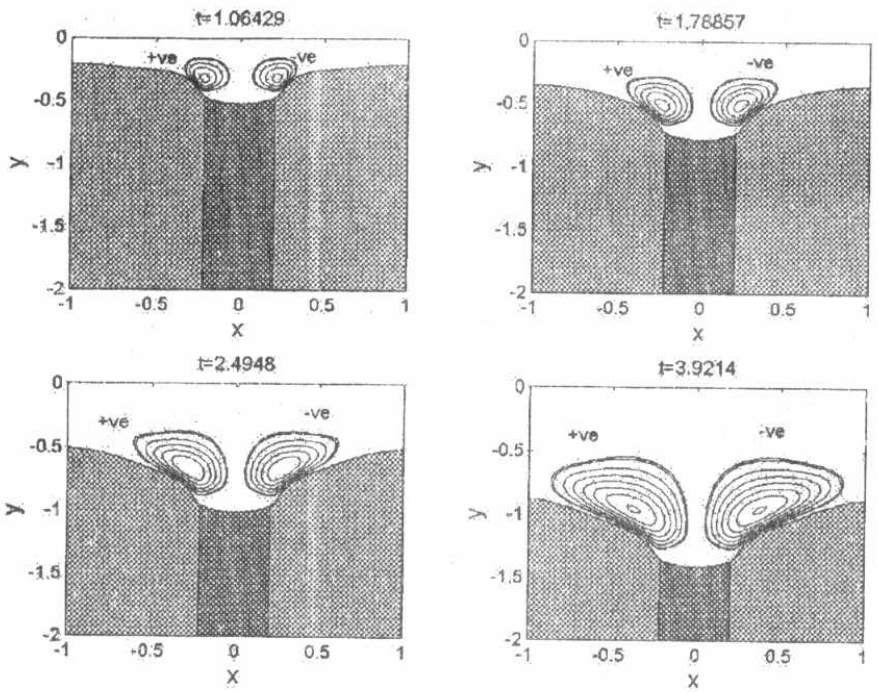

Fig. 12. The axial velocity component for the variable density model (2) at different times for $\theta=7, \beta=7, P_{e}=6, D_{a g}=5^{*} 10^{6}, P=10, M=0.02$. 